\title{
Dosimetric Characterization of a Novel Surface Collimator With Tungsten Functional Paper for Electron Therapy
}

\author{
YOSHIHIRO KAWAI ${ }^{1}$, MIKOTO TAMURA ${ }^{2}$, MORIKAZU AMANO ${ }^{1}$, \\ TAKESHI KAMOMAE ${ }^{3}$ and HAJIME MONZEN ${ }^{2}$ \\ ${ }^{1}$ Department of Radiology, Fujieda Municipal General Hospital, Shizuoka, Japan; \\ ${ }^{2}$ Department of Medical Physics, Graduate School of Medical Sciences, Kindai University, Osaka, Japan; \\ ${ }^{3}$ Department of Therapeutic Radiology, Graduate School of Medicine, Nagoya University, Aichi, Japan
}

\begin{abstract}
Background/Aim: The aim of the study was to investigate the dosimetric characteristics of electron beams with a tungsten functional paper (TFP) surface collimator. Materials and Methods: The circular field of $6.0 \mathrm{~cm}$ diameter was created with the TFP collimator put on the phantom. Depth and lateral dose profiles for 4 and $6 \mathrm{MeV}$ electron beams were obtained. The characteristics of lateral dose profile, treatment diameter as width over $90 \%$ of the dose, and penumbra as width of the off-axis positions from $80 \%$ to $20 \%$ dose levels were evaluated. Results: Compared to the lead collimator, the TFP collimator generated higher surface doses, the treatment diameters were increased from 42.8 to $48.6 \mathrm{~mm}$ and from 40.0 to $41.4 \mathrm{~mm}$, and the penumbras were reduced from 15.0 to $9.6 \mathrm{~mm}$ and from 16.4 to $13.0 \mathrm{~mm}$ for 4 and $6 \mathrm{MeV}$ electron beams, respectively. Conclusion: The TFP surface collimator can provide an excellent dose distribution compared to the conventional lead collimator.
\end{abstract}

Conventional electron therapy requires a lead collimator attached on the applicator (1-3). However, it can lead to a larger penumbra with a lateral dose spread over a wider area. Although the penumbra may be minimized by placing the collimator on the surface of the body of a patient, this is highly discouraged due to its toxicity and heavy weight of lead (4). Tungsten resin was also introduced to overcome these problems of the lead shield (5); however, it was not widely accepted because of its high price. Meanwhile, tungsten functional paper (TFP), newly developed in Japan, has radiation shielding ability with paper properties, so it is

Correspondence to: Hajime Monzen, Ph.D., Department of Medical Physics, Graduate School of Medical Sciences, Kindai University, 377-2 Ohnohigashi, Osakasayama, Osaka 589-8511, Japan. Tel: +81 723660221, Fax: +81 723657161, e-mail: hmon@med.kindai.ac.jp

Key Words: Electron therapy, dosimetric characterization, tungsten functional paper (TFP), surface collimator. easy to cut, fold, and paste to other materials. Monzen et al. have evaluated its shielding abilities with $\mathrm{X}$ and gamma rays in the diagnostic region $(6,7)$. Inada et al. have showed the usefulness of the TFP as a radiation shielding undergarment for patients with permanent prostate brachytherapy (8). In addition, several have been published on the use of TFP in electron radiotherapy applications (9-11). Fujimoto et al. have evaluated the shielding performance of the TFP with 4, 6 , and $9 \mathrm{MeV}$ electron beams and measured lateral dose profiles when the TFP was employed as a square collimator on the surface of a phantom, showing that the dose profile had a peak near the field edge due to scattering from the collimator (9).

Herein, their study was extended and dose distributions for a circular irradiation field were measured. Dosimetric comparisons were performed between the TFP collimator on a phantom surface and the conventional lead collimator on an applicator. The dosimetric parameters were evaluated as follows: 1. treatment diameter defined as the width over $90 \%$ of the dose, 2. penumbra based on the lateral dose fall-off between the off-axis position at $80 \%$ dose level and that at $20 \%$ dose level, and 3. treatment volume given at over $90 \%$ of the dose. This work is the first report that compares detailed dose characteristics including the treatment volume between the TFP and the lead collimator.

\section{Materials and Methods}

Tungsten functional paper (TFP). TFP was manufactured by Toppan Printing Co., Ltd. (Tokyo, Japan) as a sheet-shaped shielding material having a thickness of $0.3 \mathrm{~mm}$ and containing $90 \%$ tungsten powder by weight. The element ratios of the TFP (mol\%) were $\mathrm{H}$ : $24.2 \%$, C: $40.4 \%$, O: $20.2 \%$, and $\mathrm{W}: 15.2 \%$. The properties of the TFP is well explained in the literature $(6,7,9)$. The TFP was piled up to a total thickness of approximately $1 \mathrm{~cm}$ to use as surface collimator.

Equipment. The linear accelerator Infinity (Elekta AB, Stockholm, Sweden) was employed for electron beams. The three-dimensional 
scanning water phantom MP3-M (PTW, Freiburg, Germany), and the micro diamond detector TM60019 (PTW, Freiburg, Germany) were used for measuring the PDD. The Gafchromic film EBT3 (Ashland, KY, USA) was used with the water-equivalent solid phantom Tough Water Phantom, WD3005 (Kyoto Kagaku, Kyoto, Japan) for measuring dose distributions. Film analysis was performed by the film scanner, ES-G11000 (Epson, Nagano, Japan), and DD-Analysis system version 10.55 (R-TECH, Tokyo, Japan) at least $24 \mathrm{~h}$ after irradiation with a resolution of $150 \mathrm{dpi}$ at the red channel. A calibration curve, film optical density versus dose, was determined for a range from 0 to $300 \mathrm{cGy}$. The low melting-point lead MCP96 (Elekta AB, Stockholm, Sweden) was used as a reference material having a mass density of $9.85 \mathrm{~g} / \mathrm{cm}^{3}$ and a thickness of $1.6 \mathrm{~cm}$. The element ratios of the low melting-point lead $(\mathrm{mol} \%)$ were $\mathrm{Bi}: 52.5 \%, \mathrm{~Pb}: 32.0 \%$, and $\mathrm{Sn}: 15.5 \%$. The electron applicator size was $10 \times 10 \mathrm{~cm}^{2}$. Throughout this measurement, a dose of $200 \mathrm{MU}$ was delivered to the phantoms.

Measurements of PDD and dose distribution. Figure 1a shows a schematic measurement diagram of the PDD by moving a micro diamond detector in a circular field having a diameter of $6 \mathrm{~cm}$ at a source to surface distance (SSD) of $100 \mathrm{~cm}$ with energies of 4 and $6 \mathrm{MeV}$. The circular field was created by either TFP placed on the surface of a water phantom with the support of thin wires or a lead collimator placed on the applicator. The PDDs were compared between the TFP and lead collimator for each electron energy.

Figure 1b depicts another measurement diagram of the 2dimentional (2D) dose distribution in parallel to the electron beam axis under the identical beam and collimator conditions as above. The EBT3 film was inserted between the two phantom blocks on the beam's central axis, and the measured 2D dose distributions were compared between the TFP and lead collimator. The lateral dose profile was also obtained from the $2 \mathrm{D}$ dose distribution.

The depths of maximum dose $\left(d_{\max }\right), 95 \%$ dose $\left(d_{95}\right), 90 \%$ dose $\left(d_{90}\right), 80 \%$ dose $\left(d_{80}\right)$, and $50 \%$ dose $\left(d_{50}\right)$ were compared between the TFP and lead collimator. Further comparisons of treatment diameters defined as the width over $90 \%$ of the dose and penumbra based on the lateral dose fall-off of the off-axis position of $80 \%$ dose level and that of $20 \%$ dose level at depth of $90 \%$ dose $\left(d_{90}\right)$ between the TFP and lead collimator were performed. Lastly, a ratio of the treatment volume given at least $90 \%$ dose $\left(V_{90 \%}\right)$ for the TFP to the lead collimator was calculated. The $V_{90 \%}$ calculation was based on the $90 \%$ isodose contour line coordinates on the film, considering that the dose distribution was circularly symmetric in each phantom depth.

\section{Results}

Figure 2 shows the PDDs with lead (solid line) and TFP (broken line) collimators with electron energies of (a) 4 and (b) $6 \mathrm{MeV}$ by using the micro diamond detector. The TFP collimator generated higher surface doses, whereas nearly identical depth dose curves were obtained beyond the depth of maximum dose for each energy. Table I compares the values of $\mathrm{d}_{\max }, d_{95}, d_{90}, d_{80}$, and $d_{50}$ between the TFP and the lead collimator under the same circular field of $6 \mathrm{~cm}$ diameter for 4 and $6 \mathrm{MeV}$ electrons. The $\mathrm{d}_{\max }$ and $d_{95}$ for the TFP collimator were slightly smaller than that for the lead collimator, while the $d_{90}, d_{80}$ and $d_{50}$ were almost equal.
Table I. Depths of maximum $\left(d_{\max }\right), 95 \%\left(d_{95}\right), 90 \%\left(d_{90}\right), 80 \%\left(d_{80}\right)$, and $50 \%\left(d_{50}\right)$ doses for TFP and lead collimators with micro diamond detector.

\begin{tabular}{lcccc}
\hline & \multicolumn{2}{c}{$4 \mathrm{MeV}$} & \multicolumn{2}{c}{$6 \mathrm{MeV}$} \\
\cline { 2 - 5 } & Lead & TFP & Lead & TFP \\
\hline$d_{\max }(\mathrm{mm})$ & 9.0 & 8.0 & 14.0 & 13.0 \\
$d_{95}(\mathrm{~mm})$ & 10.9 & 10.6 & 17.5 & 16.8 \\
$d_{90}(\mathrm{~mm})$ & 11.8 & 11.8 & 19.1 & 18.8 \\
$d_{80}(\mathrm{~mm})$ & 13.2 & 13.2 & 21.2 & 20.9 \\
$d_{50}(\mathrm{~mm})$ & 16.2 & 16.2 & 25.6 & 25.4 \\
\hline
\end{tabular}

Table II. Treatment diameters and penumbras for TFP and lead collimators.

\begin{tabular}{lcccc}
\hline & \multicolumn{2}{c}{$4 \mathrm{MeV}$} & \multicolumn{2}{c}{$6 \mathrm{MeV}$} \\
\cline { 2 - 5 } & Lead & TFP & Lead & TFP \\
\hline Treatment diameter (mm) & 42.8 & 48.6 & 40.0 & 41.4 \\
Penumbra (mm) & 15.0 & 9.6 & 16.4 & 13.0 \\
\hline
\end{tabular}

Figures 3 and 4 show the 2D dose distributions and lateral dose profiles at $\mathrm{d}_{90}$ of the TFP and lead collimator for 4 and $6 \mathrm{MeV}$ beams, respectively. In 2D dose distributions, the isodose lines were ranged from $10 \%$ to $90 \%$, in steps of $10 \%$ of the maximum dose, filled with increasingly light color. Table II shows the comparisons of treatment diameters and penumbras between the TFP and lead collimator. Larger treatment diameter was obtained by the TFP collimator compared to the lead collimator for each electron energy. The penumbras of the TFP collimator were lower 5.4 and 3.4 $\mathrm{mm}$ compared to the lead collimator for 4 and $6 \mathrm{MeV}$ beams, respectively. The ratios of $V_{90 \%}$ for the TFP collimator to lead collimator were 2.01 and 2.39 for 4 and $6 \mathrm{MeV}$ electron beams, respectively. The $V_{90 \%}$ with the TFP collimator was increased by a factor of two or more compared to that with the lead collimator.

\section{Discussion}

In this study, the dose characteristics for the circular irradiation field of the TFP collimator were examined. This work indicated that the dose distributions of the TFP could be beneficial in clinical electron radiotherapy.

The central-axis surface dose for the TFP collimator was larger compared to the lead collimator as shown in Figure 2. The increased central-axis surface dose for the TFP collimator might be due to much smaller distance between the collimator and the phantom surface, thereby, possibly 

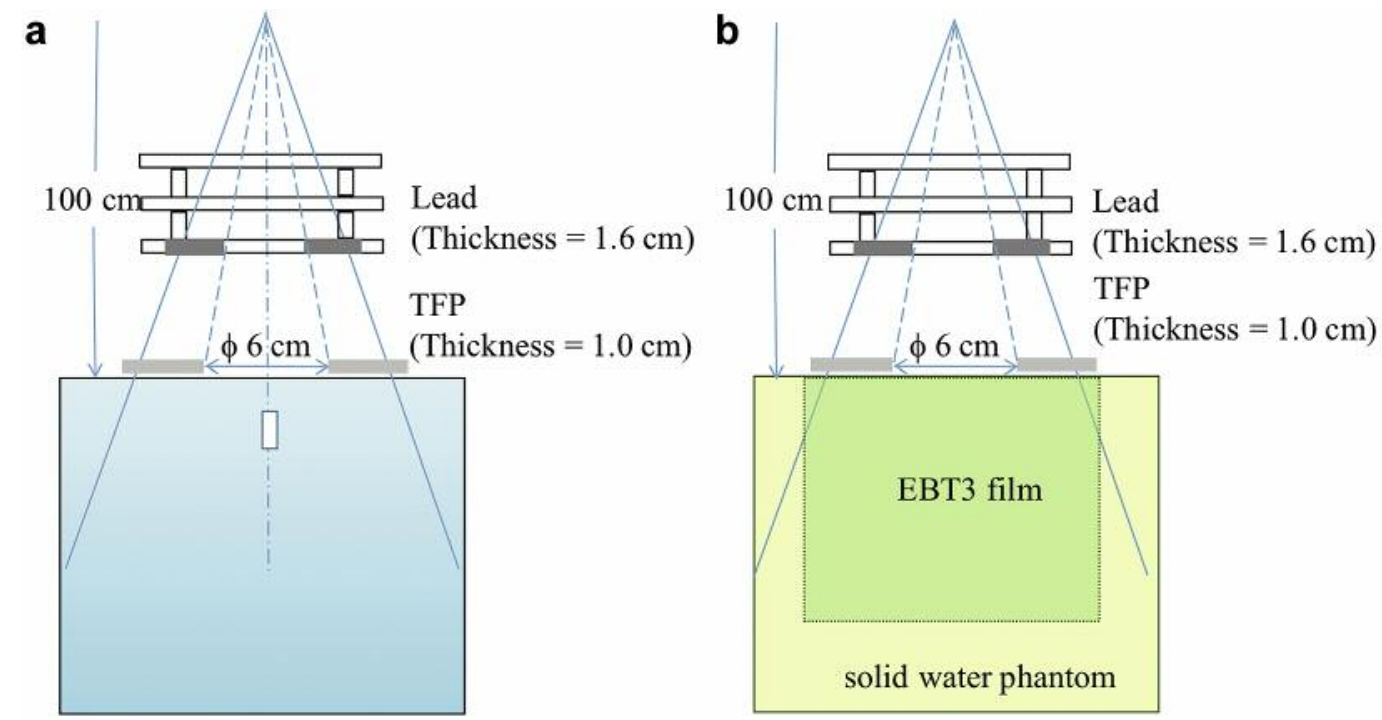

Figure 1. Schematic measurement diagrams. (a) A schematic measurement diagram of the percent depth dose (PDD) by a micro-diamond detector for a circular field of $6 \mathrm{~cm}$ diameter at a source to surface distance (SSD) of $100 \mathrm{~cm}$ with energies of 4 and $6 \mathrm{MeV}$. The circular field was created by either a tungsten functional paper (TFP) placed on the surface of a water phantom with the support of thin wires, and a lead collimator placed on the applicator. The thicknesses of the TFP and the lead were 1 and $1.6 \mathrm{~cm}$, respectively. $(b)$ A schematic measurement diagram of the 2-dimensional (2D) dose distribution of parallel to the electron beam axis under the identical beam and collimator conditions as above. An EBT3 film was inserted between the two phantom blocks, and the $2 D$ dose distributions were measured.

a

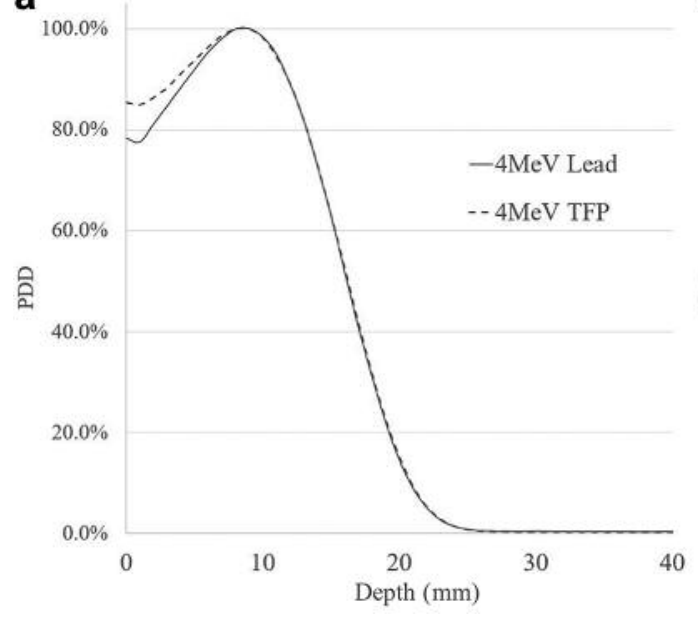

b

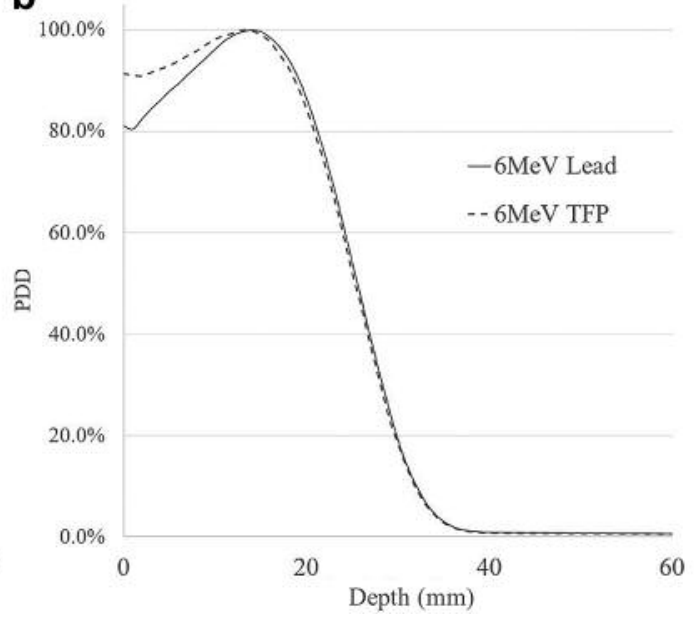

Figure 2. The PDDs with lead and TFP collimators with electron energies of (a) 4 and (b) $6 \mathrm{MeV}$. The TFP collimator generated higher surface doses, whereas nearly identical depth dose curves were obtained beyond the depth of maximum dose for each energy.

allowing more low-energy backscattered electrons from the TFP collimator edge to reach the phantom surface. Larger treatment volume could be provided by the TFP collimator compared to the lead collimator as shown in Figures 3 and 4. The much smaller distance between the TFP collimator and surface of the phantom might cause the sharp penumbra, compared to the lead collimator, and the larger treatment diameter for the TFP collimator was caused by the scattered electrons from the edge of the TFP collimator $(9,10)$. For those reasons, the $\mathrm{V}_{90 \%}$ ratio of the TFP collimator to the lead collimator was large for 4 and $6 \mathrm{MeV}$ electron beams. 

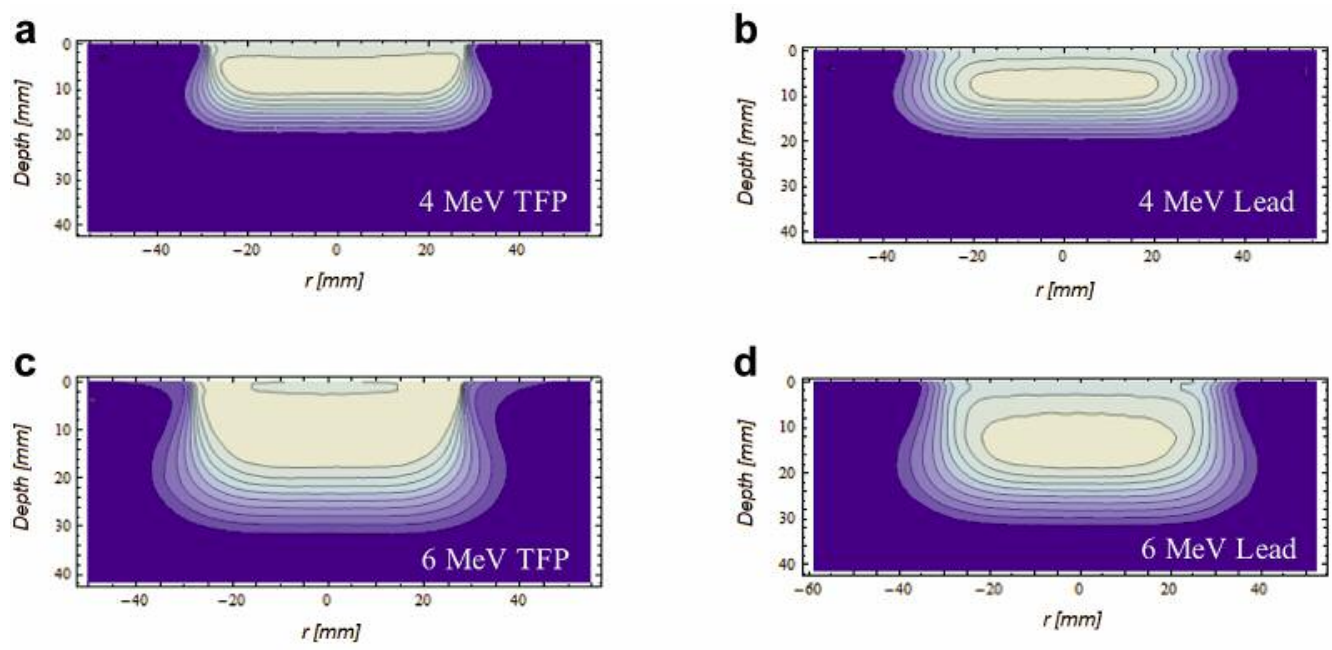

Figure 3. The 2D dose distributions measured by the EBT3 film under four different conditions: (a) $4 \mathrm{MeV}$ with TFP, (b) 4 MeV with lead, (c) 6 $\mathrm{MeV}$ with TFP, and (d) $6 \mathrm{MeV}$ with lead. The isodose line levels ranged from 10\% to $90 \%$ in steps of 10\% of the maximum dose, filled with increasingly light color.
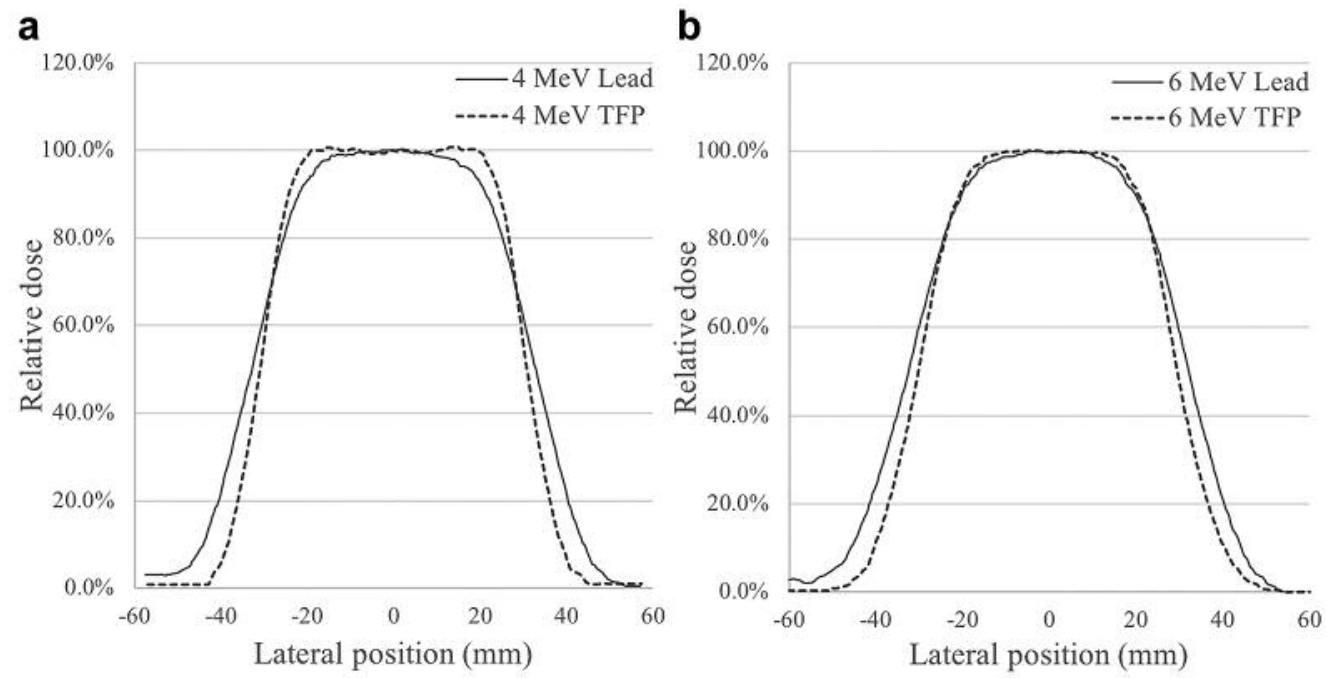

Figure 4. The lateral dose profiles at depth of $90 \%$ dose obtained from $2 D$ dose distributions with lead and TFP collimators with electron energies of (a) 4 and (b) $6 \mathrm{MeV}$. The treatment diameters with TFP collimator were larger than those of lead collimator. In addition, the penumbras with TFP collimator were sharper than those of lead collimator.

Generally, electron radiotherapy requires a bolus to increase the surface dose, however, the bolus may lead to less surface dose due to an air gap between the patient and the bolus $(12,13)$. We have confirmed that TFP collimator results in shaper penumbra as well as sufficiently higher surface dose without using a bolus, and therefore, the air gap problem can be avoided.

Among the limitations of this study is the fact that the electron energies of only 4 and $6 \mathrm{MeV}$ were employed with a TFP collimator having a thickness of $1 \mathrm{~cm}$, which may not be enough for higher energy electrons. On the other hand, the PDD curve may be more affected if the thickness of the TFP is enhanced. In addition, a field diameter of only $6 \mathrm{~cm}$ was employed and further investigations need to be performed by varying the field size, the TFP thickness and the electron energies. Future studies should also include Monte Carlo calculations to clarify detailed energy deposition physics on the surface dose resulting from the TFP collimator. In addition, studies should examine whether the TFP surface collimator can be adapted to the round contours of a patient's body without the air gap. 


\section{Conclusion}

The TFP surface collimator can provide an excellent dose distribution of high surface dose and sharp penumbra compared to the conventional lead collimator in electron radiotherapy.

\section{Conflicts of Interest}

Hajime Monzen has a consultancy agreement with, and financial interest in, TOPPAN PRINTING CO., LTD, Tokyo.

\section{Authors' Contributions}

Concept and design: YK, MA, and HM, Measurements: YK and MA, Data analysis: YK, MT, and MA, Manuscript preparation: YK, MT, MA, TK, and HM. All authors read and approved the final manuscript.

\section{Acknowledgements}

This work was supported by JSPS KAKENHI grant numbers 16K09027, 19K08211, and 19K17213.

\section{References}

1 Giarratano JC, Duerkes RJ and Almond PR: Lead shielding thickness for dose reduction of 7- to $28 \mathrm{MeV}$ electrons. Med Phys 2(6): 336-337, 1975. PMID: 811969. DOI: 10.1118/ 1.594205

2 Khan FM, Moore VC and Levitt SH: Field shaping in electron beam therapy. Br J Radiol 49(586): 883-886, 1976. PMID: 824008. DOI: $10.1259 / 0007-1285-49-586-883$

3 Khan FM, Werner BL and Deibel FC: Lead shielding for electrons. Med Phys 8(5): 712-713, 1981. PMID: 7290024. DOI: 10.1118/1.594841

4 Kijima K, Monzen H, Matsumoto K, Tamura M and Nishimura Y: The shielding ability of novel tungsten rubber against the electron beam for clinical use in radiation therapy. Anticancer Res 38(7): 3919-3927, 2018. PMID: 29970513. DOI: 10.21873/ anticanres. 12677

5 Tajiri M, Sunaoka M, Fukumura A and Endo M: A new radiation shielding block material for radiation therapy. Med Phys 31(11): 3022-3023, 2004. PMID: 15587655. DOI: 10.1118/1.1809767
6 Monzen H, Kanno I, Fujimoto T and Hiraoka M: Estimation of the shielding ability of a tungsten functional paper for diagnostic X-rays and gamma rays. J Appl Clin Med Phys 18(5): 325-329, 2017. PMID: 28656739. DOI: $10.1002 / \mathrm{acm} 2.12122$

7 Monzen H, Tamura M, Shimomura K, Onishi Y, Nakayama S, Fujimoto T, Matsumoto K, Hanaoka K and Kamomae T: A novel radiation protection device based on tungsten functional paper for application in interventional radiology. J Appl Clin Med Phys 18(3): 215-220, 2017. PMID: 28422397. DOI: 10.1002/acm2. 12083

8 Inada $\mathrm{M}$, Monzen $\mathrm{H}$, Matsumoto $\mathrm{K}$, Tamura $\mathrm{M}$, Minami T, Nakamatsu $\mathrm{K}$ and Nishimura Y: A novel radiation-shielding undergarment using tungsten functional paper for patient with permanent prostate brachytherapy. J Radiat Res 59(3): 333-337, 2018. PMID: 29659976. DOI: 10.1093/jrr/rry030

9 Fujimoto T, Monzen H, Nakata M, Okada T, Yano S, Takakura T, Kuwahara J, Sasaki M, Higashimura $\mathrm{K}$ and Hiraoka M: Dosimetric shield evaluation with tungsten sheet in 4, 6, and 9 MeV electron beams. Phys Med 30(7): 838-842, 2014. PMID: 24953537. DOI: 10.1016/j.ejmp.2014.05.009

10 Tamura M, Monzen H, Kubo K, Hirata M and Nishimura Y: Feasibility of tungsten functional paper in electron grid therapy: A Monte Carlo study. Phys Med Biol 62(3): 878-889, 2017. PMID: 28072577. DOI: 10.1088/1361-6560/62/3/878

11 Kamomae T, Monzen H, Kawamura M, Okudaira K, Nakaya T, Mukoyama T, Miyake Y, Ishihara Y, Itoh Y and Naganawa S: Dosimetric feasibility of using tungsten-based functional paper for flexible chest wall protectors in intraoperative electron radiotherapy for breast cancer. Phys Med Biol 63(1): 015006, 2017. PMID: 29083315. DOI: 10.1088/1361-6560/aa96cf

12 Sharma SC and Johnson MW: Surface dose perturbation due to air gap between patient and bolus for electron beams. Med Phys 20(2): 377-378, 1993. PMID: 8497226. DOI: 10.1118/1.597079

13 Kong M and Holloway L: An investigation of central axis depth dose distribution perturbation due to an air gap between patient and bolus for electron beams. Australas Phys Eng Sci Med 30(2): 111-119, 2007. PMID: 17682400. DOI: 10.1007/ BF03178415
Received March 20, 2019

Revised May 13, 2019

Accepted May 14, 2019 